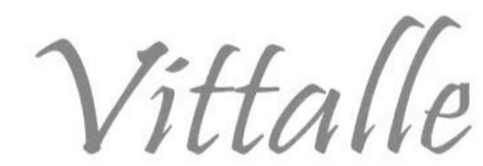

\title{
Ambiente e conceitos de computação em nuvem: implementação de um banco de dados para uso no segmento hospitalar
}

\author{
Thiago Gonçalves dos Santos Martins ${ }^{\mathrm{a}, b^{*}}$, Tulio Cezar Alves de Souza ${ }^{\mathrm{c}}$, Márcio \\ Alexandre Marques ${ }^{\mathrm{c}}$, Augusto Voltaire do Nascimento ${ }^{\mathrm{d}}$, Paulo Schor ${ }^{\mathrm{a}}$ \\ aUniversidade Federal de São Paulo, Departamento de Oftalmologia, São Paulo, SP, Brasil \\ bUniversidade de Coimbra, Faculdade de Medicina, Coimbra, Portugal \\ 'Departamento de Engenharia de Controle e Automação, Instituto de Ciência e Tecnologia de Sorocaba, \\ Universidade Estadual Paulista - UNESP, Sorocaba, SP, Brasil \\ ${ }^{\mathrm{d}}$ Hospital do Servidor Público Estadual de São Paulo (HFMO), Serviço de Pneumologia, São Paulo, SP, Brasil
}

\begin{abstract}
Histórico do Artigo:
Recebido em:

15/07/2020

Aceito em:

03/09/2020

Palavras-chave:

Aplicação em

biomedicina; uso de

computação em

nuvem; solução de

infraestrutura;

inovação em

tecnologia de saúde;

big data

Keywords:

Application in biomedicine; cloud computing use; infrastructure solution; health IT innovation; big data

RESUMO

Uma ferramenta foi desenvolvida utilizando um banco de dados em nuvem para auxiliar profissionais de saúde. Dessa forma, uma aplicação foi implementada para o uso hospitalar com a finalidade de colaborar nas atividades laborais de médicos, enfermeiros e todos os demais envolvidos no cotidiano de um estabelecimento da área da saúde, inclusive os pacientes. Escolheu-se o banco de dados da Oracle como base do desenvolvimento da ferramenta. A implementação do software permitiu a otimização da rotina de trabalho dos profissionais de saúde. Além disso, como a aplicação está baseada em nuvem pública, o seu uso e acesso são facilitados através de diferentes dispositivos como laptops, tablets e smartphones. Isso também permite ao hospital compartilhar o sistema com outros hospitais, clínicas e laboratórios parceiros sem grandes dificuldades, com o objetivo de unificar a base de informações e trabalhar de forma coordenada para obter melhores resultados.

Environment and cloud computing concepts: implementation of a database for use in the hospital segment

\section{ABSTRACT}

A tool was developed using a cloud database to assist healthcare professionals. Thus, an application was implemented for hospital use in order to collaborate in the work activities of doctors, nurses and everyone else involved in the daily life of a health care establishment, including patients. The Oracle database was chosen as the basis for the development of the tool. The software implementation allowed the optimization of the health professionals' work routine. In addition, as the application is based on a public cloud, its use and access are facilitated through different devices such as laptops, tablets and smartphones. This also allows the hospital to share the system with other partner hospitals, clinics and laboratories without major difficulties, in order to unify the information base and work in a coordinated way to obtain better results.
\end{abstract}

\section{Introdução}

No Brasil, tem aumentado a utilização de prontuários eletrônicos nos serviços de atenção básica, com o objetivo de integrar os sistemas de informatização de saúde municipais e estaduais ao sistema nacional. Dessa forma, todas as informações dos pacientes estarão disponíveis on-line e com isso, os dados do prontuário eletrônico podem ser acessados de qualquer lugar do território nacional, disponibilizando detalhes de atendimentos realizados evitando assim, a repetição de exames, além de reduzir

\footnotetext{
*Autor correspondente: thiagogsmartins@yahoo.com.br (Martins T.G.S.)
} 
custos por evitar a emissão de receitas em duplicata e consequentemente, retirada de medicamentos em duplicata.

As nuvens computacionais têm sido uma solução na área de saúde para a grande quantidade de dados armazenados e gerados (1-5). A computação em nuvem proporciona um modelo de armazenamento de dados em larga escala com a flexibilidade necessária para o processamento desses dados. Essa tecnologia apresenta a eliminação da necessidade de o estabelecimento de saúde possuir um servidor em suas instalações onde um sistema teria que ser instalado, redes internas de acesso a esses servidores e a sua consequente necessidade de manutenção e equipe de suporte. Entretanto, alguns problemas surgem com essa nova tecnologia, como os relacionados à segurança e privacidade dos dados pessoais, médicos e científicos $(6,7)$.

A computação em nuvem permite um modelo de acesso a uma quantidade de recursos computacionais configuráveis (por exemplo, redes, servidores, armazenamento, aplicações e serviços) que podem ser rapidamente provisionados e liberados. A tecnologia de computação em nuvem, de maneira geral, nada mais é que a disponibilização de dados, aplicações e acessos por meio de uma plataforma que mantenha estes dados hospedados fora do computador ou dispositivo que está acessando essas informações. Dependendo da modalidade e metodologia de implementação utilizadas para a computação na nuvem, esses dados podem estar em uma rede fechada, mista ou totalmente aberta, no caso da internet (8).

As nuvens computacionais podem documentar ameaças emergentes à saúde pública em tempo real. Um exemplo é o Smarter Public Health Prevention System (SPHPS). O SPHPS fornece relatórios em tempo real de potenciais ameaças à saúde pública para decisores, através do uso de plataformas móveis (celular, smartphone e tablet), visando promover e incentivar comportamentos saudáveis nas comunidades (9-11).

Dessa forma, foi desenvolvida uma ferramenta computacional, utilizando computação em nuvem, para uso hospitalar com o objetivo de auxiliar as atividades laborais de médicos, enfermeiros e demais envolvidos no cotidiano de um estabelecimento da área da saúde, inclusive dos pacientes. Implementou-se ainda a possibilidade de que o paciente também possua um acesso para consultar o resultado de seus exames, bem como, alterar suas informações de contato em seu cadastro básico.

A proposta desse trabalho foi então, verificar as necessidades de um hospital e comprovar a viabilidade técnica da aplicação a ser implementada, sem realizar testes com uma grande quantidade de dados e nem mesmo em um cenário com todos os usuários reais. Assim, o objetivo foi desenvolver uma ferramenta utilizando um banco de dados em nuvem para auxiliar profissionais de saúde.

\section{Materiais e métodos}

No desenvolvimento desta ferramenta, optou-se pelo emprego da tecnologia em nuvem, ou seja, a aplicação conta com um grande diferencial do ponto de vista de disponibilidade, uma vez que, por estar construída num banco de dados rodando em modelo de Plataforma Como Serviço (PaaS - Platform as a Service) e em nuvem pública do fornecedor Oracle, disponibiliza-se na nuvem um ambiente com capacidade de desenvolvimento e criação de aplicações, integrações, códigos e rotinas para o cliente.

Neste modelo possibilita-se, por exemplo, adaptar e desdobrar aplicações para uso comercial. Aqui o cliente não administra a infraestrutura sobre a qual a plataforma e os códigos estarão sendo desenvolvidos e rodando. Neste caso, a responsabilidade e custos envolvidos com o desempenho e uptime cabem à Oracle e podem estar vinculados ao 
contrato firmado entre o hospital e o fornecedor.

Nesse sentido, para implementação da aplicação, o prontuário eletrônico foi desenvolvido abordando os seguintes conteúdos: adendos da internação, evolução diária do paciente, prescrição, encaminhamento a outros profissionais, exames, cuidados da enfermagem, administração da prescrição, cuidados fisioterápicos, controle de dados dietéticos, informações de assistência social e a classificação internacional de doenças (CID). Escolheu-se o banco de dados da Oracle como base do desenvolvimento da ferramenta hospitalar. O aplicativo foi desenvolvido para atender a necessidade de todos os usuários do atendimento de saúde (Quadro1).

Quadro 1 - Relação de atividades esperadas para cada perfil.

\begin{tabular}{|c|c|}
\hline Perfil dos Usuários & Atividades esperadas na aplicação \\
\hline Recepcionistas & $\begin{array}{l}\text { Consultar pacientes cadastrados, verificar seu histórico, } \\
\text { registrar novos pacientes, cadastrar um novo atendimento e } \\
\text { alterar informações básicas de cadastro dos pacientes. }\end{array}$ \\
\hline Médicos & $\begin{array}{l}\text { Verificar os pacientes em atendimento, bem como seu histórico } \\
\text { no hospital. Para o atendimento atual, consultar todo o } \\
\text { prontuário e ter a possiblidade de incluir informações, como } \\
\text { adendo de internação, evolução diária do paciente, prescrição } \\
\text { médica, solicitação de exames e registro de encaminhamentos. } \\
\text { Além disso, um médico deve ser capaz de alterar o status do } \\
\text { atendimento para um paciente e registrar as doenças e suspeitas } \\
\text { usando o Cadastro Internacional de Doenças (CID). }\end{array}$ \\
\hline Enfern & $\begin{array}{l}\text { Consultar todo o prontuário do paciente, incluindo as } \\
\text { informações adicionadas pelos médicos e demais profissionais, } \\
\text { como também adicionar dois tipos de informação ao prontuário } \\
\text { do paciente (evolução dos cuidados da enfermagem e } \\
\text { informações da administração da prescrição). }\end{array}$ \\
\hline Fisioterapeutas & $\begin{array}{l}\text { Consultar todo o prontuário do paciente, incluindo as } \\
\text { informações adicionadas pelos médicos e demais profissionais, } \\
\text { da mesma forma, adicionar a evolução diária dos cuidados } \\
\text { fisioterápicos ao prontuário do paciente. }\end{array}$ \\
\hline Nutricionistas & $\begin{array}{l}\text { Consultar todo o prontuário do paciente, incluindo as } \\
\text { informações adicionadas pelos médicos e demais profissionais } \\
\text { e do mesmo modo, adicionar as informações do controle } \\
\text { dietético do paciente. }\end{array}$ \\
\hline $\begin{array}{l}\text { Agentes de Serviço } \\
\text { Social }\end{array}$ & $\begin{array}{l}\text { Consultar todo o prontuário do paciente, incluindo as } \\
\text { informações adicionadas pelos médicos e demais profissionais } \\
\text { bem como adicionar as informações de assistência aos } \\
\text { problemas sociais e/ou familiares do paciente. }\end{array}$ \\
\hline Técnicos & $\begin{array}{l}\text { Consultar todos os pedidos de exame realizados nos prontuários } \\
\text { e adicionar status e resultados destes exames. }\end{array}$ \\
\hline Pacientes & $\begin{array}{l}\text { Consultar e alterar seus dados de contato, como também, } \\
\text { consultar e realizar download dos exames realizados quando do } \\
\text { atendimento no hospital. }\end{array}$ \\
\hline
\end{tabular}


A interface de acesso do programa foi desenvolvida contendo usuário e senha, onde o sistema realiza a identificação de acordo com o perfil de acesso e apresenta ao usuário as opções pertinentes apenas ao seu perfil.

Neste ponto, um grande diferencial da aplicação desenvolvida é que, por estar disponível em internet pública, o programa permite o acesso fora do ambiente hospitalar utilizando computadores pessoais, bem como dispositivos móveis como tablet ou smartphone e assim, os médicos conseguem constantemente opinar e consultar informações de casos, atendimentos e pacientes.

Uma vez concebido o perfil de cada profissional e suas atividades esperadas na aplicação, implementou-se a estrutura das interfaces a serem acessadas como campos, dados e tabelas a serem criadas no banco de dados e consequentemente, o fluxo de navegação esperado em cada perfil.

Uma "massa" de dados fictícia foi criada com 15 médicos, oito enfermeiros, três nutricionistas, três fisioterapeutas, quatro recepcionistas, três agentes de serviço social, três técnicos e 30 pacientes. Todos os nomes e informações para elaboração dessa base de dados foi feita de maneira aleatória, com a finalidade de testar este trabalho e não são condizentes com nomes e informações de pessoas reais.

Após a aplicação ter sido idealizada, "desenhada" e a base de dados criada, solicitouse um banco de dados na nuvem ao fornecedor, no caso a Oracle Corporation. Por meio do site APEX (https://apex.oracle.com/) foi possível então, ter acesso à ferramenta Application Express (APEX) da Oracle, rodando sobre um Banco de Dados na nuvem exclusivo para este trabalho. A Figura 1 mostra um exemplo de uma arquitetura de sistema onde o Oracle APEX se conecta ao mundo externo e a outros sistemas proprietários.

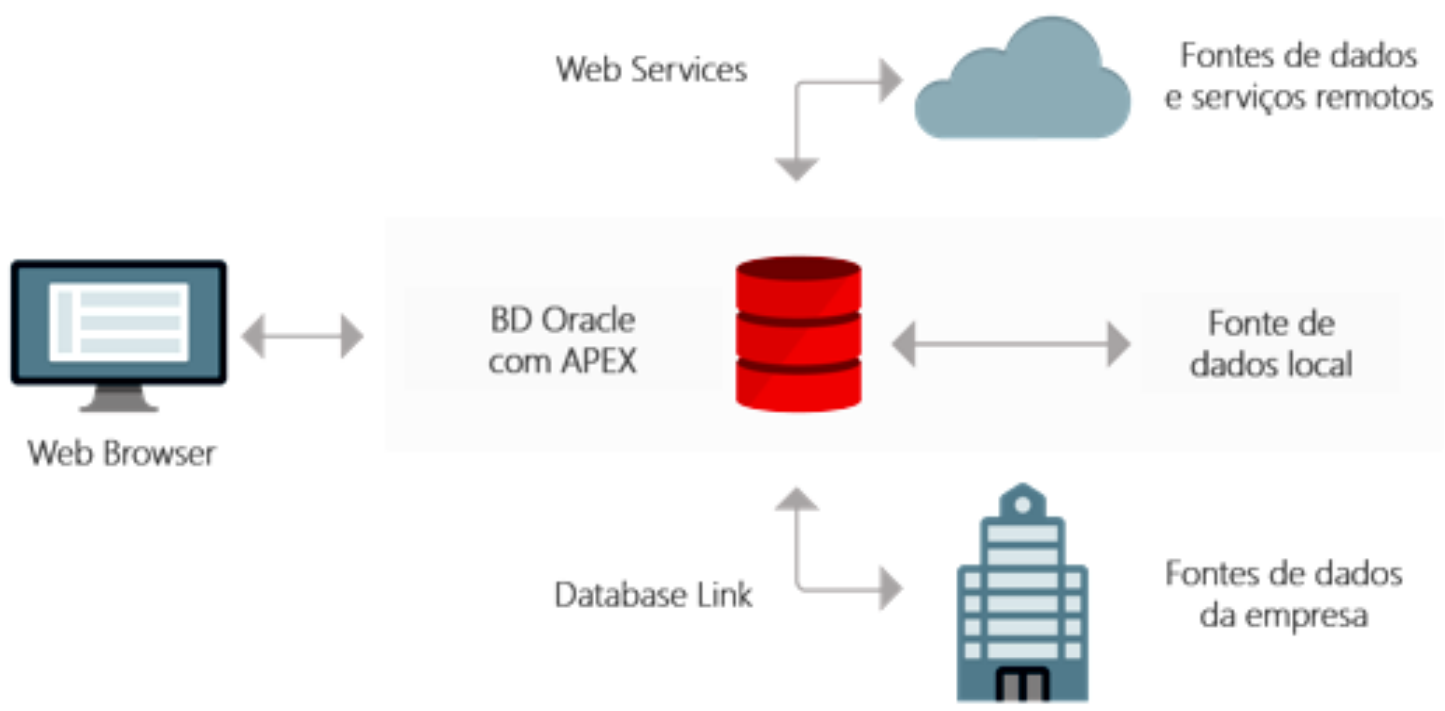

Figura 1 - Exemplo de uma arquitetura de sistema com ligações a fontes de dados (12).

Posteriormente, foram criadas as tabelas no banco de dados e a inclusão da "massa" de dados previamente preparada para cada uma delas. As tabelas e demais dados puderam ser carregados de forma massiva por meio da ferramenta APEX.

Uma vez que o banco de dados já estava estruturado com todas as tabelas, dados, "gatilhos" e informações, pode-se então passar para a criação da aplicação propriamente dita e das interfaces onde os usuários acessariam as informações deste banco de dados de acordo com cada perfil de acesso. A criação das interfaces da aplicação na 
ferramenta APEX foi feita de acordo com uma interface de desenvolvimento, mostrada na Figura 2.

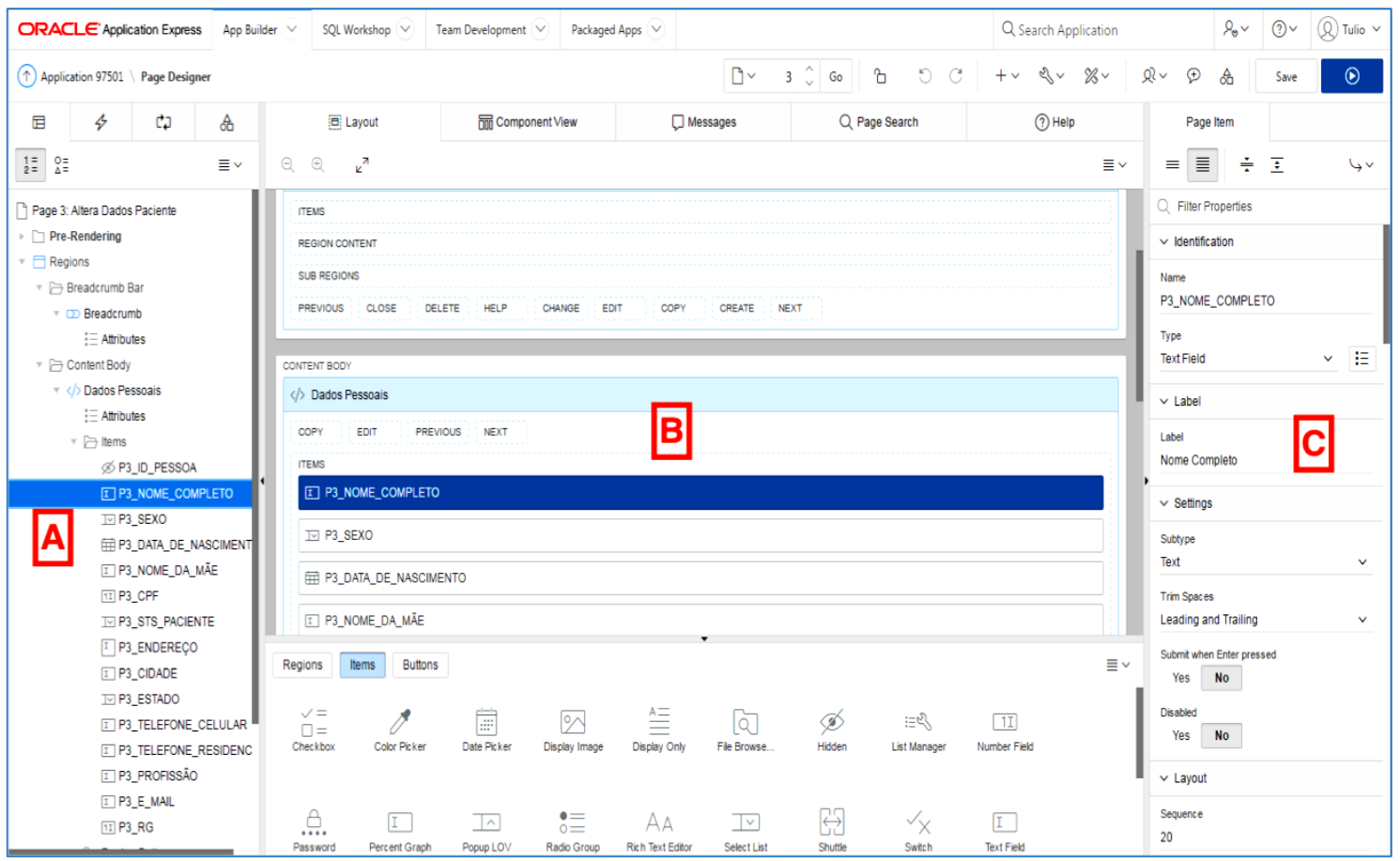

Figura 2 - Exemplo de interface da ferramenta APEX.

Na Figura 2, pode-se observar que a interface de desenvolvimento está dividida em três grandes áreas. Na primeira, à esquerda identificada como "A", ficam dispostos todos os itens, campos e regiões que fazem parte desta interface. Na segunda, ao centro ("B") é trabalhada a disposição e o layout destes campos e regiões. Na terceira, à direita da interface ("C"), ficam as propriedades de um campo ou região selecionado. É nesta última área que são definidos os comportamentos e as características de cada área ou região da interface que está sendo desenvolvida.

As interfaces que visualizam informações do banco de dados são majoritariamente constituídas de regiões denominadas relatórios. São elas as responsáveis por exibir os dados armazenados no banco de dados em uma determinada tabela ou em construir a visualização compondo dados advindos de mais de uma tabela do banco de dados.

A Figura 3 mostra um exemplo de arquitetura multicamada semelhante a que foi utilizada na implementação da ferramenta, onde à esquerda, encontra-se o que está na camada externa (usuário acessando a aplicação via internet), no meio a Zona Desmilitarizada - DMZ (criação das interfaces ("telas") e modelo de dados na aplicação propriamente dita) e na direita o que está exatamente no banco de dados - as tabelas, views e pacote de dados. 


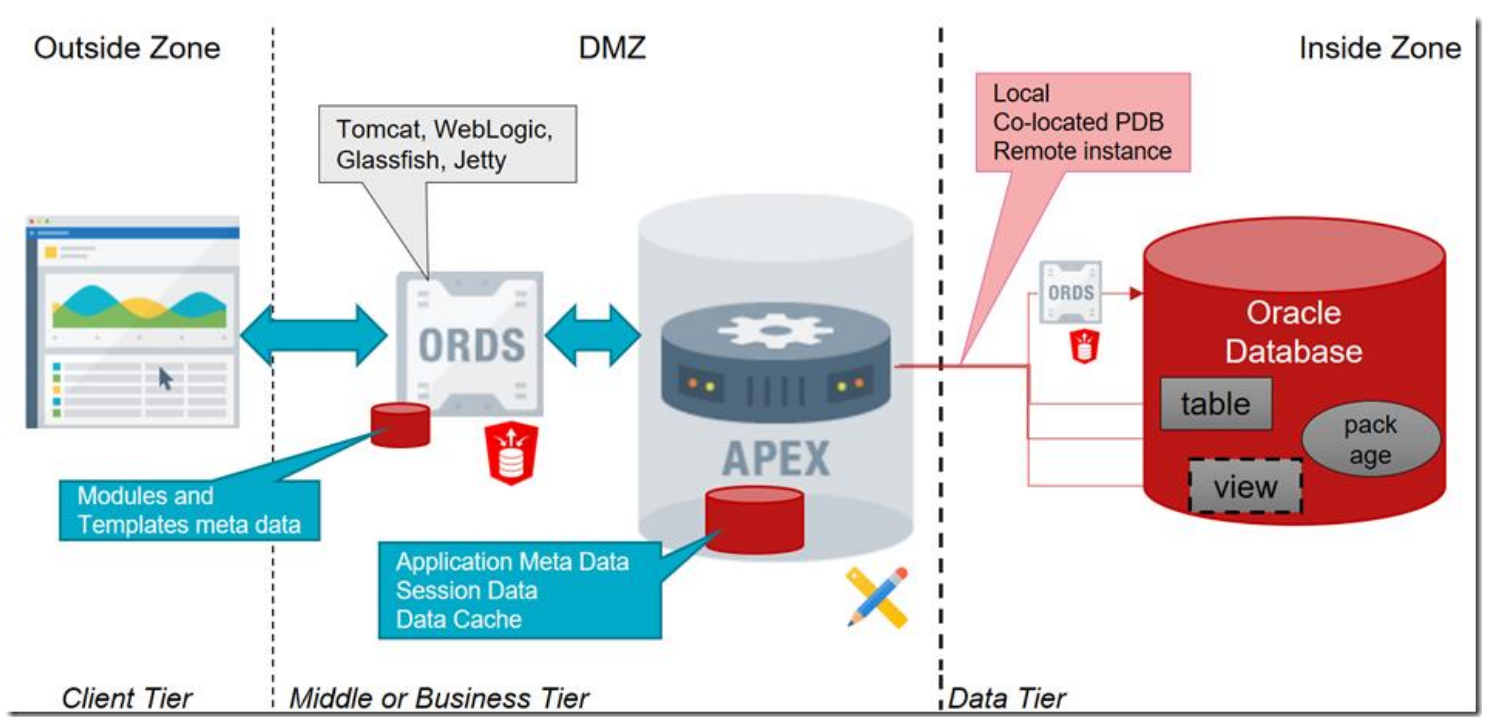

Figura 3 - Arquitetura multicamadas para a abordagem mais comum para aplicativos APEX (13).

\section{Resultados}

Como resultado da implementação da aplicação, obteve-se um sistema completo onde os oito perfis de usuários podem cumprir suas funções no dia a dia hospitalar. Ao acessar a aplicação, o usuário é redirecionado à primeira interface, denominada "interface de acesso".

Assim que o usuário digita seu nome de usuário e sua senha, o sistema faz a validação e informa se as credenciais estão corretas. Em caso negativo, uma mensagem de erro é exibida. Caso tenha fornecido as informações corretas, ele é redirecionado à interface de boas-vindas que apresenta o nome de usuário, o grupo de usuários ao qual ele pertence e um menu de navegação que mostra as interfaces onde o usuário que está acessando a aplicação tem permissão de atuar. De acordo com o grupo de usuários, um conjunto diferente de interfaces é exibido neste menu.

A seguir, são apresentados exemplos de acesso através do perfil de um médico e de um paciente, visto que o sistema funciona de forma semelhante para os outros perfis.

\section{Perfil do usuário: Médicos}

Quando um médico acessa a aplicação, ele terá disponível na interface de boas-vindas, outros dois botões: Home Médico e Atendimentos.

O primeiro, Home Médico, vai apresentar a ele a lista de pacientes internados atualmente na enfermaria, bem como a contagem de internados. Essa interface pode ser vista na Figura 4. 


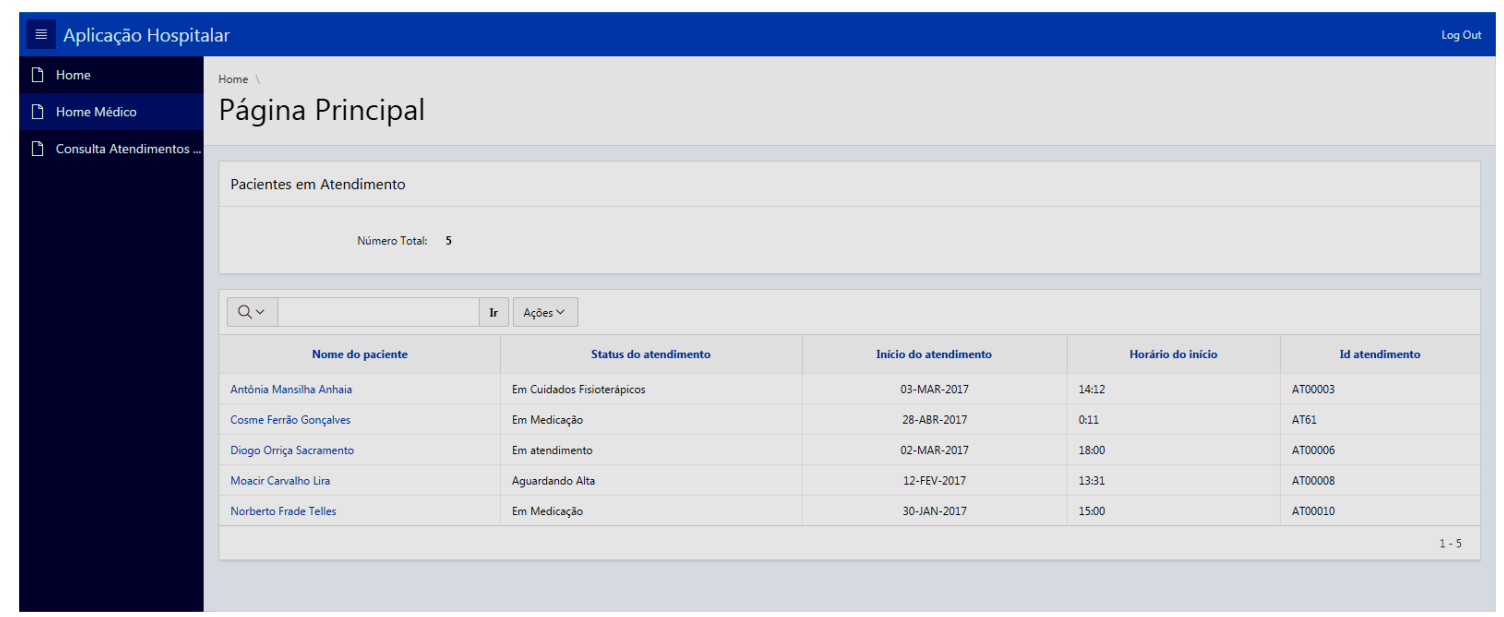

Figura 4 - Lista de pacientes internados mostrada ao médico.

Caso o médico deseje acessar o histórico de um paciente internado, basta fazer um clique no nome deste paciente e ele será redirecionado para a "tela" seguinte.

Nesta nova interface, quando o médico clica no nome de um paciente para um atendimento ativo, ele acessa a interface de detalhes do prontuário, onde pode interagir, aba por aba (Alterar Status, Adicionar Adendo da Internação, etc.) na totalidade das informações do paciente. Essa interface é mostrada na Figura 5.

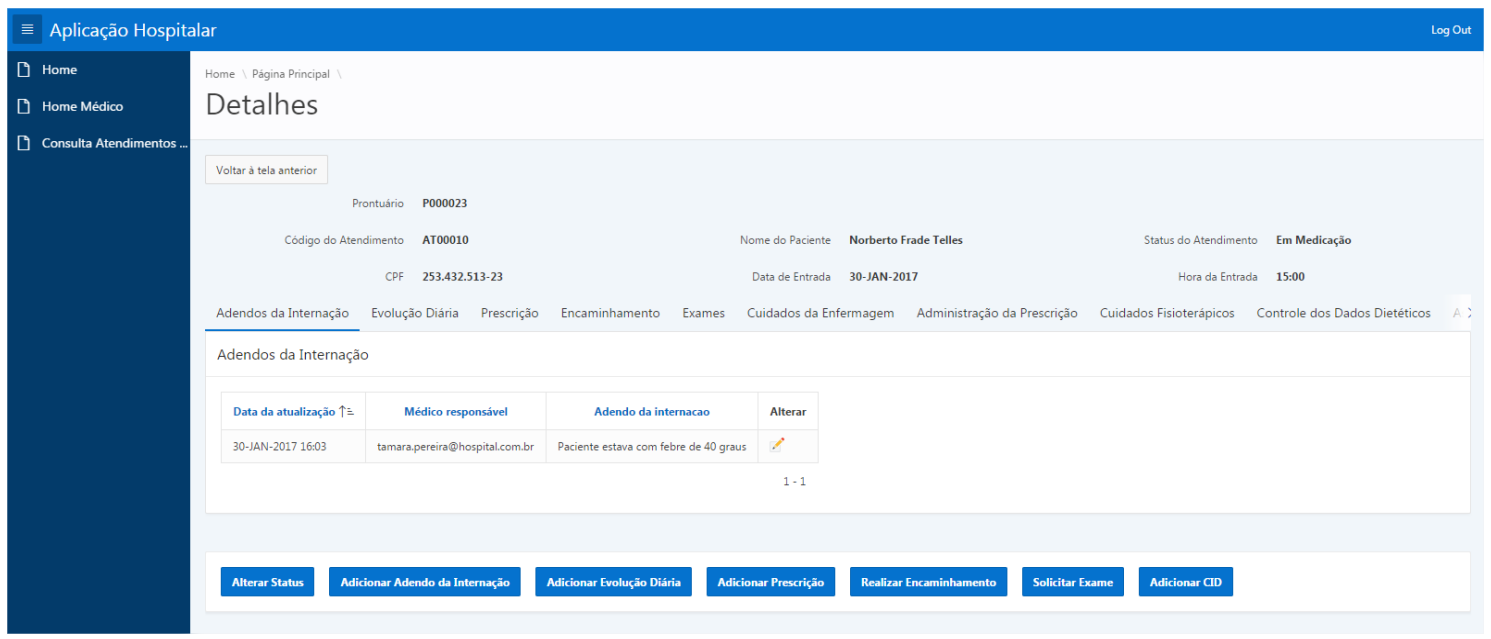

Figura 5 - Detalhes do prontuário de atendimento vista pelo médico.

Nesta interface (Figura 5), o médico pode acessar as ações correspondentes ao seu perfil no prontuário do paciente, mas em cada uma das abas ele também pode visualizar o que os outros profissionais, como enfermeiros e nutricionistas adicionaram ao prontuário.

\section{Perfil do usuário: Pacientes}

Quando os pacientes acessam a ferramenta e o sistema os identifica, eles irão encontrar no seu menu duas opções: "Minhas Informações Pessoais" e "Resultados dos Meus Exames". Ao acessar o primeiro item, o paciente é então direcionado para uma interface que contém as suas informações pessoais.

Caso o paciente note alguma inconsistência em algum dos seus dados, ele pode clicar no botão "Alterar Minhas Informações" para alterar as próprias informações e então, o sistema libera os campos para ele realizar as alterações. $\mathrm{O}$ sistema não permite a edição 
de campos como nome, CPF e data de nascimento para garantir a segurança e privacidade dos dados. O paciente ainda pode consultar, no segundo item do menu de acesso ("Resultados de Exames"), os exames realizados em seus atendimentos e efetuar o download de cada um dos exames cujos resultados já estejam disponíveis. Caso o exame ainda não esteja disponível, o botão de download não aparece para esse usuário.

Todas as funcionalidades da aplicação desenvolvida para o gerenciamento de um banco de dados foram testadas para todos os perfis de usuários descritos no Quadro 1. Os testes foram realizados por diferentes profissionais da área de saúde e pelos desenvolvedores do sistema (Engenheiro e Físico (da área de computação)). Destaca-se ainda, que a escolha da tecnologia em nuvem para a implementação da aplicação é um diferencial que também facilitou o seu uso e acesso através de diferentes dispositivos como laptops, tablets e smartphones, visto que essa tecnologia possibilitou o acesso através de uma simples conexão à internet.

Apesar dos testes terem sido feitos com uma "massa" de dados pequena, os resultados corroboram o fato de que a ferramenta pode ser implementada em um ambiente hospitalar e utilizada fora dele, sendo, portanto, um facilitador no dia a dia dos médicos, dos enfermeiros e de todos os demais envolvidos no cotidiano de um estabelecimento da área da saúde, incluindo os pacientes.

\section{Discussão}

O sistema possui um registro eficiente e contempla informações para sete tipos de perfis de usuários que rotineiramente trabalham com os atendimentos. Já o perfil de paciente, tem permissão apenas para realizar alterações nas suas informações pessoais. A ferramenta utiliza uma interface simples, mas completa, que garante aos profissionais acesso integral de todas as informações. As permissões de acesso criadas na solução funcionaram corretamente e da maneira esperada para todos os perfis, garantindo que nenhum usuário acessasse conteúdos e ações que estivessem fora da sua área de atuação. Esse procedimento garante a confiabilidade e a segurança das informações presentes no sistema.

Outro diferencial da aplicação desenvolvida é que, por estar disponível em internet pública, médicos constantemente precisam opinar e consultar informações de casos, atendimentos e pacientes. Mesmo quando estão fora do hospital ou ainda em trânsito, a aplicação construída permite ao médico realizar este acesso remotamente por meio de qualquer dispositivo que tenha uma conexão à internet. Isso facilita a colaboração e o engajamento das equipes. Nesse sentido a utilização da aplicação é possível em qualquer dispositivo, como tablets e smartphones, o que permite ao profissional da área de saúde realizar atualização do prontuário a partir de qualquer lugar, inclusive quando estiver visitando o leito do paciente. Essa característica evita que médicos, enfermeiros e os demais profissionais precisem realizar a anotação de informações no leito do paciente e posteriormente, dirigir-se a um computador específico para realizar o registro sistemático das informações.

A característica dessa solução em nuvem permite ainda que o hospital deixe de usar servidores, redes e equipes dedicadas a manter uma infraestrutura própria. Dessa maneira, a administração poderia direcionar mais recursos ao atendimento de pacientes e reduzir o custo de propriedade no uso da tecnologia.

O uso de armazenamento de informações em nuvem possui limitações que precisam ser controladas como o impedimento de processar dados clínicos devido aos requisitos de privacidade e da legislação de diversos países. Algumas plataformas recentes implementaram mecanismos para aumentar o nível de privacidade e segurança dos 
dados clínicos $(14,15)$. Para contornar esse problema, o software tem a criação de esquema de autenticação e permissões de acesso baseado em grupos, de modo que cada profissional acesse apenas as áreas e ações que são pertinentes ao seu perfil.

Outra limitação do uso dos dados na nuvem é a transferência de grandes volumes de dados ou informações. Muitas vezes, o tempo necessário para transmitir um grande volume de dados excede em muito o tempo para processá-los (16). Alguns estudos têm tentado contornar esse problema com a utilização da computação através de streaming ou armazenamento prévio de grandes bases de dados na própria nuvem, minimizando a necessidade de transferência de dados, e deste modo, otimizando o desempenho das aplicações a serem executadas na nuvem $(16,17)$. Outra restrição é a necessidade de conexão permanente, dificultando o acesso em locais com conexão de baixa velocidade, o que gera problemas de conexão entre o usuário e a nuvem, já que nem todos os programas podem estar disponíveis remotamente (18). O desenvolvimento dessa tecnologia também aumenta a necessidade de capacitação de cientistas de dados na área de medicina, com objetivo de analisar essa grande quantidade de dados que possam ser gerados (19-22).

Os dados clínicos dos prontuários eletrônicos oferecem grandes oportunidades para o avanço na área biomédica, contudo devemos sempre manter a proteção dos dados dos pacientes. $\mathrm{O}$ uso de dados em nuvem demonstrou ser uma solução adequada para o armazenamento e processamento de uma grande quantidade de dados, podendo ter diversas aplicações na saúde pública, buscando uma redução de custos e uma melhora ao atendimento da população. Isso pode mudar o panorama de países como o Brasil, onde o número de hospitais e clínicas com uso de tecnologia é restrito. Os sistemas estão presentes principalmente em processos administrativos e de faturamento, e raramente no centro das atividades clínicas.

Isso permite ampliar as atividades dedicadas ao cuidado aos pacientes, ao invés de usá-los em atividades burocráticas. Uma documentação de dados mais eficaz tem potencial de minimizar o trabalho manual, aumentando a adesão de profissionais de saúde e a qualidade dos dados obtidos, gerando assim, dados mais confiáveis e de qualidade. Além disso, a tecnologia escolhida para a realização desta aplicação permite ainda a sua expansão de maneira simples caso seja identificada alguma necessidade de alteração ou inclusão de novas funcionalidades, visto que esta aplicação não foi implementada com linguagem de programação proprietária ou exclusiva.

\section{Limitações do estudo}

Considerando que o objetivo desse trabalho foi verificar e comprovar a viabilidade técnica da aplicação a ser implementada, novos estudos clínicos longitudinais são necessários para testar o uso da plataforma com um maior número de profissionais de saúde e permitir futuras adaptações conforme a necessidade da especialidade médica e da característica de cada estabelecimento de saúde. Apesar disso, os testes realizados com um número pequeno de dados não comprometeram a validade da ferramenta desenvolvida.

Bancos de dados autônomos já estão disponíveis e permitem atualização automática sem intervenção humana, utilizando tecnologia de inteligência artificial.

\section{Conclusões}

O desenvolvimento da aplicação permitiu a simulação da otimização da rotina de trabalho dos profissionais de saúde, garantindo qualidade no uso das informações e dos 
dados. Os testes realizados por diferentes profissionais da área de saúde e pelos desenvolvedores do sistema comprovaram a viabilidade técnica para a implementação da ferramenta, bem como o seu uso e acesso através de diferentes dispositivos móveis conectados à internet. Como a aplicação está baseada em tecnologia em nuvem pública, isso permite ao hospital compartilhar o sistema com outros hospitais, clínicas e laboratórios parceiros, com o objetivo de unificar a base de informações e trabalhar de forma coordenada para obter melhores resultados.

\section{Ajuda financeira: não}

\section{Conflito de interesses: não}

\section{Referências}

1. Raghupathi W, Raghupathi V. Big data analytics in healthcare: promise and potential. Health Inf Sci Syst 2014; 2(1): 3 .

2. Costa FF. Big data in biomedicine. Drug discovery today 2014; 19(4): 433-40.

3. Reshef DN, Reshef YA, Finucane HK, Grossman SR, McVean G, Turnbaugh PJ, et al. Detecting novel associationsin large data sets. Science 2011; 334(6062): 1518-24.

4. Chin L, Andersen JN, Futreal PA. Cancer genomics: from discovery science to personalized medicine. Nat Med 2011; 17(3): 297-303.

5. Dai L, Gao X, Guo Y, Xiao J, Zhang Z. Bioinformatics clouds for big data manipulation. Biol Direct 2012; 7(1): 43.

6. Marx V. Biology: the big challenges of big data. Nature 2013; 498(7453): 255-60.

7. O'Driscoll A, Daugelait J, Sleator RD. 'Big data', Hadoop and cloud computing in genomics. J Biomed inform 2013; 46(5): 774-81

8. Mell P, Tim G. The NIST definition of cloud computing. Communic ACM. 2010; 53(6): 50.

9. Jalali A, Olusegun AO, Bell CM. Leveraging cloud computing to address public health disparities: an analysis of the SPHPS. Online J Pub Health inform. 2012; 4(3).

10. Piette JD, Mendoza-Avelares MO, Ganser M, Mohamed M, Marinec N, Krishnan S. A preliminary study of a cloud computing model for chronic illness self-care support in an underdeveloped country. Am J Prev Med 2011; 40(6): 629-32.

11. Kuo AM. Opportunities and challenges of cloud computing to improve health care services. J Med Internet Res 2011; 13(3): e67.

12. Simões, R, Cardoso, C. Oracle application express: introdução ao APEX. BI4all: turning data into insights [Blog]. Lisboa, 25 ago. 2017. Disponível em: https://www.bi4all.pt/noticias/blog/oracleapplication-express-introducao-ao-apex/ Acesso em: 17 ago. 2020.

13. Jellema, L. Oracle APEX: the low-code and low-cost application middle tier. AMIS Technology Blog. Nieuwegein, 1, nov. 2018. Disponível em: https:/technology.amis.nl/2018/11/01/oracle-apexthe-low-code-and-low-cost-application-middle-tier/ Acesso em: 14 ago. 2020.

14. Bessani A, Brandt J, Bux M, Cogo V, Dimitrova L, Dowling J, et al. BiobankCloud: a platform for the secure storage, sharing, and processing of large biomedical data sets.

15. Proceedings of the 1st Int. In: Workshop on Data Management and Analytics for Medicine and Healthcare (DMAH 2015); 2015 Sept 4; Kohala Coast. Hawaii, US.

16. DNAnexus Inc. Compliance with HIPAA, CLIA, dbGaP, EU Privacy, and ISO 27001 on DNAnexus. [cited 2015 Set 9]. Available from: https://www.dnanexus.com/papers/Compliance_White_Paper.pdf

17. Issa SA, Kienzler R, El-Kalioby M, Tonellato PJ, Wall D, Bruggmann R, Abouelhoda M. Streaming support for data intensive cloud-based sequence analysis. Biomed Res Int 2013; 2013: 791051.

18. Langille MG, Eisen JA. BioTorrents: a file sharing service for scientific data. PLoS One 2010; 5(4): e10071

19. Arutynov, V. V. Cloud Computing: its history of development, modern state, and future 
considerations. Scientific and Technical Information Processing 2012; 1(3): 173-178.

20. Margolis R, Derr L, Dunn M, Huerta M, Larkin J, Sheehan J, et al. The National Institutes of health's big data to knowledge (BD2K) initiative: capitalizing on biomedical big data. J Am Med Inform Assoc 2014; 21(6): 957-8.

21. Martins TGDS, Costa ALFA, Martins TGDS. Big Data use in medical research. Einstein (Sao Paulo) 2018; 16(3): eED4087.

22. Alves M, Forschini RA, Schor P. Using Big Data in eye research to answer important scientific questions. Arq Bras Oftalmol 2019; 82(3): V-VI.

23. Perakakis N, Yazdani A, Karniadakis GE, Mantzoros C. Omics, big data and machine learning as tools to propel understanding of biological mechanisms and to discover novel diagnostics and therapeutics. Metabolism 2018; 87: A1-A9. 